\title{
Electrospray Ionization Quadrupole Ion-Mobility Time-of-Flight Mass Spectrometry as a Tool to Distinguish the Lot-to-Lot Heterogeneity in N-Glycosylation Profile of the Therapeutic Monoclonal Antibody Trastuzumab
}

\author{
Carola W. N. Damen, ${ }^{a}$ Weibin Chen, ${ }^{\mathrm{b}}$ Asish B. Chakraborty, \\ Mike van Oosterhout, ${ }^{\mathrm{c}}$ Jeffrey R. Mazzeo, ${ }^{\mathrm{b}}$ John C. Gebler, ${ }^{\mathrm{b}}$ \\ Jan H. M. Schellens, ${ }^{\text {,e }}$ Hilde Rosing, ${ }^{\text {a }}$ and Jos H. Beijnen ${ }^{\mathrm{a}, \mathrm{d}}$ \\ ${ }^{a}$ Department of Pharmacy and Pharmacology, Slotervaart Hospital/The Netherlands Cancer Institute, \\ Amsterdam, The Netherlands \\ ${ }^{\mathrm{b}}$ Biopharmaceutical Sciences, Waters Corporation, Milford, Massachusetts, USA \\ ${ }^{c}$ Waters Chromatography B.V., Etten-Leur, The Netherlands \\ d Beta faculty, Department of Pharmaceutical Sciences, Division of Biomedical Analysis, Utrecht University, \\ Utrecht, The Netherlands \\ ${ }^{\mathrm{e}}$ Department of Medical Oncology, Antoni van Leeuwenhoek Hospital/The Netherlands Cancer Institute, \\ Amsterdam, The Netherlands
}

\begin{abstract}
Monoclonal antibodies are typically glycosylated at asparagine residues in the Fc domain, and glycosylation heterogeneity at the FC sites is well known. This paper presents a method for rapid analysis of glycosylation profile of the therapeutic monoclonal antibody trastuzumab from different production batches using electrospray quadrupole ion-mobility time-of-flight mass spectrometry (ESI-Q-IM-TOF). The global glycosylation profile for each production batch was obtained by a fast LC-MS analysis, and comparisons of the glycoprofiles of trastuzumab from different lots were made based on the deconvoluted intact mass spectra. Furthermore, the heterogeneity at each glycosylation site was characterized at the reduced antibody level and at the isolated glycopeptide level. The glycosylation site and glycan structures were confirmed by performing a time-aligned-parallel fragmentation approach using the unique dual-collision cell design of the instrument and the incorporated ion-mobility separation function. Four different production batches of trastuzumab were analyzed and compared in terms of global glycosylation profiles as well as the heterogeneity at each glycosylation site. The results show that each batch of trastuzumab shares the same types of glycoforms but relative abundance of each glycoforms is varied. (J Am Soc Mass Spectrom 2009, 20, 2021-2033) (C) 2009 American Society for Mass Spectrometry
\end{abstract}

$\mathrm{M}$ onoclonal antibodies (MABs) are an upcoming group of disease-modifying drugs. Their efficacy results from specificity and inhibition of the target antigen and from biological activities (effector functions) activated by immune complexes formed. MABs are used to treat cancer, auto-immune diseases, and systemic infections [1]. Trastuzumab (Herceptin) is a humanized monoclonal immunoglobulin $\gamma$-1 (IgG1) antibody directed against the HER2/neu receptor, which is over-expressed in about $25 \%$ of all breast cancer patients [2, 3].

An IgG1 molecule consists of two identical heavy chains and two identical light chains that are connected to each other by four disulfide bonds. Each light chain

Address reprint requests to Dr. Carola W. N. Damen, Department of Pharmacy and Pharmacology, Slotervaart Hospital/The Netherlands Cancer Institute, Louwesweg 6, 1066 EC Amsterdam, The Netherlands. E-mail: carola.damen@slz.nl additionally contains two intra-chain disulfide bonds and each heavy chain four intra-chain disulfide bonds leading to 16 disulfide bonds for the entire protein. Asparagine at position 297 in the heavy chains is N-glycosylated and heterogeneity in this glycosylation profile is common for IgG antibodies $[4,5]$. The most common glycan structures for IgG possess zero, one, or two terminal galactose $(G)$ residues with or without a fucose $(\mathrm{F})$ and are defined as G0, G0F, G1F, and G2F [6, 7].

All antibody therapeutics that are currently licensed have been produced by mammalian cell culture, utilizing Chinese hamster ovary $(\mathrm{CHO})$ cells or mouse NSO or Sp2/0 plasma cell lines [4]. Trastuzumab is produced in CHO cells. The efficacy of a MAB is critically dependent on appropriate post-translational modifications, and each production vehicle produces different profiles as post-translation modifications show species, tissue, and site specificity. In vitro studies have established 
that essential effector functions are dependent on appropriate glycosylation of the antibody molecule [4]. Additionally glycosylation can influence clearance and immunogenicity of the MAB [5, 8]. Therefore, FDA requires analytical characterization of MABs [9] and a consistent human-type glycosylation of the MABs. Elucidation of the produced glycosylation structure is thus pivotal for the pharmaceutical industry.

Several techniques have been published about the analysis of the glycosylation profile. In early years the MABs were deglycosylated and analyzed with, e.g., ${ }^{1} \mathrm{H}$ NMR [10], sodium dodecyl sulfate-polyacrylamide gel electrophoresis (SDS-PAGE) [11], hydrophobic interaction chromatography [11], and high-pH anion-exchange chromatography with pulsed amperometric detection (HPAEC-PAD) [12]. Radio labeling of the released glycans, and analyzing them by a combination of paper electrophoresis and gel-filtration chromatography [13], with normal phase high-performance liquid chromatography (HPLC) $[14,15]$ or anion-exchange HPLC [16] coupled with a fluorescence detector have also been described. Additionally, capillary electrophoresis coupled with different detectors has been described [1719]. More recently, several methods using mass spectrometry (MS) have been developed. These analyses are performed using HPLC coupled with either electrospray ionization single quadruple mass spectrometry (ESI-MS) [20, 21], or ESI quadruple time-of-flight MS (ESI-Q-TOF-MS) [8, 22-24], or ESI orthogonal acceleration time-of-flight MS (ESI-oa-TOF-MS) [6, 25, 26]. Matrix assisted laser desorption/ionization-TOF-MS (MALDI-TOF-MS) is also a widely applied technique [14, 22, 27-29]. Most of these methods analyze the intact or reduced $\mathrm{MAB}$ or tryptic digest of MAB. The generated ESI mass spectra are deconvoluted, and the accurate masses of the found peaks are compared with the theoretical masses of MABs with the most common glycans. However, these methods are not capable of performing true structural elucidation of the glycan moieties as they only measure the masses of the subunit or peptides and compare them against the masses of known protein sequence and most common glycans.

Tandem mass spectrometry is a method of choice for structural elucidation of glycans in terms of composition, sequence, linkage, and branching. Published reports for structural elucidation generally involve an enzymatic digestion or a chemical deglycosylation step to obtain either glycopeptides or released glycans for subsequent analysis using HPLC coupled with ESI-QTOF MS, ESI-ion trap MS [30-32], ESI triple quadrupole MS [33], or linear ion trap Fourier transform MS (LTQFTMS) [34]. Nevertheless, these methods are time consuming, requiring high-resolution separation of the peptides and glycans on the HPLC system, and/or long separation time. In addition, for LC-MS analysis of released glycans, due to the low ionization efficiency and hydrophilicity imposed by the chemical nature of the oligosaccharides, derivatization is often applied leading to time consuming sample preparations.
Sinha et al. [35] compared various sample preparation schemes coupled with a HPLC-ESI-Q-TOF MS and compared two production lots of a recombinant monoclonal IgG1 antibody. Direct analysis of glycopeptides by tandem mass spectrometry (MS/MS) represents a rapid and sensitive method to obtain site-specific characterization on glycosylation. It can provide information on glycan structure, glycan attachment site, and peptide sequence within one single experiment. However, direct fragmentation of glycopeptides normally generates very complex mass spectra, which contain fragment peaks from the intact or truncated glycan chains as well as the deglycosylated peptide backbones (after the complete loss of glycan chain). This heterogeneity, together with the superimposition of various charge states, makes the MS/MS spectra interpretation rather difficult. As a result, the successful application of this approach to the site-specific glycosylation is rather limited.

In this paper, we present a LC-MS method to distinguish the lot-to-lot heterogeneity of the commercially available monoclonal antibody trastuzumab (Herceptin) by a quadrupole ion-mobility time-of-flight mass spectrometer (ESI-Q-IM-TOF-MS). Four different production batches of trastuzumab were analyzed and compared. To establish a global view of the IgG isoforms, the intact $\mathrm{MAB}$ proteins were first analyzed using on-line LC/MS. Then subunits of the intact MABs were analyzed after reduction, to confirm the heterogeneity of the glycoforms at the heavy chain of trastuzumab. On the basis of analysis of global glycoprofile, detailed characterization of the glycans was performed using a bottom-up approach on tryptic glycopeptides. The detailed analysis of the glycopeptide takes advantage of a unique dual-collision-cell design of the instrument combined with ion-mobility separations to perform a time-aligned-parallel (TAP) fragmentation of glycopeptides, in which information on the glycan sequence and the glycosylation site is simultaneously obtained. The results demonstrate the benefit of enabling dual-stage fragmentation where the independently controlled collision induced dissociation provides an increase in the information obtained for sitespecific characterization of the glycopeptide. Since the detailed characterization on the glycosylation heterogeneity can be performed on a single instrument platform and within a short time, the approach is well suited for lot-to-lot testing of production batches of MABs and for fast elucidation of the glycosylation profiles.

\section{Experimental}

\section{Chemicals and Reagents}

All chemicals were of highest purity available. Dithiothreitol (DTT), iodoacetamide (IAA), Tris- $\mathrm{HCl}$, EDTA, ammonium bicarbonate, and calcium chloride were purchased from Sigma (St. Louis, MO, USA). Guanidine$\mathrm{HCl}$ was obtained from Pierce (Rockford, IL, USA). 
Anhydrous acetonitrile was from Fisher Scientific (Pittsburgh, PA, USA). Formic acid was obtained from EM Sciences (Gibbstown, NJ, USA). Ultrapure water (18.2 M) was obtained from a Milli-Q water purifier (Millipore Corporation, Medford, MA, USA). Sequencinggrade porcine trypsin was obtained from Promega Corp. (Madison, WI, USA). RapiGest SF was obtained from Waters Corporation (Milford, MA, USA). Four different batches (B0011, B1142, B2033, and B2045) of trastuzumab (Herceptin) as a lyophilized powder were purchased from Roche (Basel, Switzerland). This pharmaceutical formulation contains additionally L-histidine hydrochloride, L-histidine, $\alpha$ - $\alpha$-trehalose dihydrate and polysorbate 20 . Trastuzumab was dissolved in water for injections according to the instruction from the manufacturer, resulting in a stock solution of $21 \mathrm{mg} / \mathrm{mL}$. The solution was diluted to $0.5 \mathrm{mg} / \mathrm{mL}$ with $50 \mathrm{mM}$ ammonium bicarbonate for intact mass analysis using ESI Q-IM-TOF MS.

\section{Reduction of Trastuzumab}

Partial reduction of the IgG1 was performed according to the following method [36, 37]: the trastuzumab stock solution $(4 \mu \mathrm{L})$ was mixed with $66 \mu \mathrm{L}$ of $3 \mathrm{M}$ Tris- $\mathrm{HCl}$ buffer ( $\mathrm{pH}$ 8.0) containing $1 \mathrm{mM}$ EDTA to obtain a working solution with $1.2 \mathrm{mg} / \mathrm{mL}$ of MAB. Two $\mu \mathrm{L}$ of $1 \mathrm{M}$ DTT (in $25 \mathrm{mM} \mathrm{NH} \mathrm{NCO}_{3}$ ) was added to the solution, and the mixture was vortex mixed briefly before incubation at $37^{\circ} \mathrm{C}$ for $20 \mathrm{~min}$. The reduction was then quickly quenched with a $2 \%$ (vol/vol) formic acid aqueous solution to a concentration of $0.11 \mathrm{mg} / \mathrm{mL}$ $(\mathrm{pH}$ 3.0). The reduced sample was prepared daily before analysis.

\section{Preparation of Tryptic Glycopeptides of Trastuzumab}

An aliquot of trastuzumab stock solution $(21 \mathrm{mg} / \mathrm{mL})$ was diluted with $8 \mathrm{M}$ urea in $400 \mathrm{mM}$ ammonium bicarbonate to obtain a solution with $2.1 \mathrm{mg} / \mathrm{mL}$ of trastuzumab. To this solution, a volume of $500 \mathrm{mM}$ EDTA in $\mathrm{H}_{2} \mathrm{O}$ was added. Trastuzumab was then reduced with $10 \mathrm{mM}$ DTT at $37^{\circ} \mathrm{C}$ for $30 \mathrm{~min}$, and alkylated with $13 \mathrm{mM}$ IAA in the dark at room temperature for $45 \mathrm{~min}$. The reduced and alkylated trastuzumab solution was further diluted by pure $\mathrm{H}_{2} \mathrm{O}$ to a concentration of $0.5 \mathrm{mg} / \mathrm{mL}$ for trypsinization. Trypsin digestion was performed overnight at $37^{\circ} \mathrm{C}$ (trypsin/ protein ratio, 1:25, wt/wt). The digest was diluted to $0.015 \mathrm{mg} / \mathrm{mL}$ with $0.1 \%$ formic acid before injection.

\section{Preparation of Released Glycans from Intact Protein of Trastuzumab}

A solution of Trastuzumab $(4.5 \mathrm{mg} / \mathrm{mL})$ was prepared by diluting the stock solution $(21 \mathrm{mg} / \mathrm{mL})$ with a solution of $0.1 \%$ (wt/vol) RapiGest in $50 \mathrm{mM}$
$\mathrm{NH}_{4} \mathrm{HCO}_{3}$. The protein sample was reduced with 10 $\mathrm{mM}$ DTT for $45 \mathrm{~min}$ and alkylated with $20 \mathrm{mM}$ iodoacetamide for $1 \mathrm{~h}$. The enzyme PNGase F was added to the sample, the solution was incubated overnight. The released glycans were extracted by following the published procedure [38], and were subsequently labeled with 2-AB using the manufacturer protocols (Sigma). Labeled glycans were purified by HILIC SPE (Waters Corp.) and dried down. Approximately $\sim 50$ pmol of labeled glycans were loaded into Waters Glycan column $(2.1 \times 150 \mathrm{~mm})$. The HILIC separation was done with $0.5 \%$ formic acid in $\mathrm{H}_{2} \mathrm{O}$ (mobile phase A) and $0.5 \% \mathrm{FA}$ in acetonitrile $(0.22 \% \mathrm{~B} / \mathrm{min}$. $)$.

\section{Mass Spectrometry}

All experiments were performed on a Waters Synapt high definition mass spectrometer (HDMS) fitted with an atmospheric pressure ionization electrospray source (Waters Corporation, Milford, MA, USA). Detailed descriptions of the instrument can be found elsewhere [37, $39,40]$. The instrument was operated in positive-ion mode with a capillary voltage of $3.0 \mathrm{kV}$ unless stated otherwise. The ion source block and nitrogen desolvation gas temperatures were set to $100^{\circ} \mathrm{C}$ and $250{ }^{\circ} \mathrm{C}$, respectively, unless stated otherwise. The desolvation gas was set to a flow rate of $250 \mathrm{~L} / \mathrm{h}$. The cone voltage was maintained at $60 \mathrm{~V}$ for intact mass analysis. The quadrupole was operated in nonresolving mode to transmit a wide $m / z$ range. Full-scan data for proteins and tryptic digests were acquired over $\mathrm{m} / \mathrm{z}$ range of 500 to 4000 or 50 to 1990, respectively. Data collection and processing were controlled by MassLynx 4.1 software (Waters Corp.). The deconvolution of ESI mass spectra of intact/reduced proteins was performed using the MaxEnt1 algorithm in the MassLynx software.

\section{Analysis of Intact Trastuzumab and Trastuzumab Subunits by LC/MS}

An ACQUITY UPLC system (Waters Corp.) equipped with a binary solvent manager, a sample manager, and a column heater was directly coupled to a Synapt HDMS mass spectrometer using a standard ESI interface for all the analyses. Reversed-phase (RP) desalting of intact and reduced antibody was performed on a phenyl desalting column, $(2.1 \times 5 \mathrm{~mm}, 20 \mu \mathrm{m}$, Waters Corp). Mobile phase A was $0.1 \%$ formic acid in water, mobile phase B was $0.1 \%$ formic acid in acetonitrile. For intact trastuzumab analysis, the column was first washed isocratically for $0.5 \mathrm{~min}$ at $5 \% \mathrm{~B}$ at a flow rate of $0.5 \mathrm{~mL} / \mathrm{min}$ to remove all the salts and other contaminants in the samples. The antibody was then eluted using a $1.5 \mathrm{~min}$ gradient $(5 \%$ to $90 \% \mathrm{~B})$ at a flow rate of $0.2 \mathrm{~mL} / \mathrm{min}$ and a column temperature of $80^{\circ} \mathrm{C}$. Following each experiment, three additional rapid gradient cycles $(0.5 \mathrm{ml} / \mathrm{min}$ and between 5 and $90 \% \mathrm{~B})$ were applied to condition the column to pre-injection state 
for next injection. The total run time for intact antibody analysis is $4 \mathrm{~min}$. For the LC/MS analysis of partially reduced trastuzumab samples, a different gradient ( $10 \%$ to $50 \%$ B in $7 \mathrm{~min}$ ) was used to achieve maximized chromatographic separation of the light and heavy chains on the desalting column. The mass spectrometer was calibrated externally using CsI (2 mg/mL) dissolved in 50\% isopropanol. Analysis of intact trastuzumab was performed with a capillary voltage of 2.0 $\mathrm{kV}$, sample cone of $60 \mathrm{~V}, \mathrm{~m} / \mathrm{z}$ range of 1000 to 4000, and mass resolution of 10,000 ( $\mathrm{V}$ mode). For trastuzumab subunits, the data were collected with a capillary voltage of $2.8 \mathrm{kV}$, sample cone of $35 \mathrm{~V}, \mathrm{~m} / \mathrm{z}$ range of 600 to 3500 , and mass resolution of 10,000. The MS ion source and desolvation temperatures were set at 100 and $350{ }^{\circ} \mathrm{C}$, respectively, for the experiments. The desolvation gas flow rate was set to $600 \mathrm{~L} / \mathrm{h}$ at the source and desolvation temperatures discussed above. All four batches of trastuzumab were analyzed by the exactly same experimental settings with the same amount of materials injected. The parameters used for MaxEnt1 deconvolution of the MS data from the heavy chain are the following: input mass $(\mathrm{m} / \mathrm{z})$ range: 995-1995; output mass range: 46,000-5,000; output data resolution: 1 $\mathrm{Da} /$ channel; model: uniform Gaussian with peak width at half height 0.6 ; minimum intensity ratios: 30\% (left and right); complete iteration: convergence.

\section{Analysis of Glycopeptides by nanoLC/MS/MS}

To illustrate the possibilities of a full structural elucidation of the glycan moieties one batch of trastuzumab was chosen (B0011). The analyses of glycopeptides from this batch were performed on the Synapt HDMS mass spectrometer coupled with a nanoAcquity (Waters Corp.) system. Tryptic peptides of trastuzumab (1.4 pmol) were separated by a reversed-phase column (Atlantis $\mathrm{C}_{18}, 3.5 \mu \mathrm{m}, 0.3 \times 100 \mathrm{~mm}$; Waters Corp.). Peptides were eluted at $10 \mu \mathrm{L} / \mathrm{min}$ with a linear gradient profile consisting of 3\% to $40 \%$ B over $60 \mathrm{~min}$, where Solvent A was water containing $0.1 \%$ (vol/vol) formic acid and Solvent B was acetonitrile containing $0.1 \%$ (vol/vol) formic acid.

The LC elution time and the $m / z$ value of glycopeptides were measured by operating the mass spectrometer in TOF mode using a method described previously [37, 41]. Briefly, as the peptides were eluted off the column, MS data were acquired in a continuous fashion by alternating low and elevated energies of the TRAP cell so all the peptides eluting from the HPLC column as well as the corresponding fragmented ions were recorded simultaneously. A scan time of $1.0 \mathrm{~s}$ was set for both low-energy (MS) data acquisition and elevatedenergy $\left(\mathrm{MS}^{\mathrm{E}}\right)$ spectra. During the low-energy scan, intact peptide mass spectra were obtained by keeping collision cell energy at $4 \mathrm{~V}$, while the multiplex fragmentation $\mathrm{MS}^{\mathrm{E}}$ spectra were obtained by ramping collision cell energies from 15 to $35 \mathrm{~V}$. Both low-energy mass spectra and elevated-energy spectra were acquired in the $m / z$ range of 50 to 1990 .

Fragmentation of glycans and glycopeptides was performed by operating the instrument in the mobilityTOF mode. The instrument was set to perform MS/MS analysis of glycopeptides at the LC elution time determined in the previous run. Each glycopeptide was sequentially selected by the quadrupole and fragmented in the TRAP cell at the energy optimized for glycan fragmentation (collision energy set at $35 \mathrm{~V}$ ). The fragment ions generated in the TRAP T-wave collision cell of Synapt HDMS were then transported into the IMS T-wave where ions were separated into individual packets on the basis of their ion mobility (combination of size, shape and charge as well as mass). The ionmobility separated fragment ions were subsequently transported through the TRANSFER T-wave collision cell in which the collision energy was alternated between two settings (low and high). When the collision energy of the TRANSFER T-wave is set at a low-energy (normally at $5 \mathrm{~V}$ ), no additional fragmentation takes place in the TRANSFER T-wave. Fragment ions observed in this scan would only represent the fragments generated in the TRAP T-wave. Thus MS/MS data recorded in this process would contain information only for the deduction of the glycan sequence of the glycopeptides. To obtain the fragmentation of peptide backbone, the collision energy of the TRANSFER Twave is increased to a higher energy (typically 60 to 100 $\mathrm{V})$ in the second scan, and the ion-mobility separated product ions are subjected to a second-generation fragmentation at which peptide backbone cleavage would occur, and thus producing data for the deduction of peptide sequence and glycosylation sites.

MS/MS data from the same precursor were combined, smoothed, background-subtracted, and deconvoluted to collapse the isotope peaks using the MaxEnt 3 program in the MassLynx software. MaxEnt3 processes a spectrum with peaks in several charge states and produces a simplified spectrum consisting of only singly charged monoisotopic peaks. The spectrum generated from this process was either interpreted manually (for glycan sequence information) or submitted to a de novo sequencing software (BioLynx) for the final peptide sequence deduction.

\section{Results and Discussion}

\section{Analysis of Intact Trastuzumab}

Figure 1a shows the charge state distribution of trastuzumab from batch B2033. When the spectrum is examined in greater detail as in Figure $1 \mathrm{~b}$ where the +53 charge state is shown, it can be observed that each charge state consists of multiple peaks. When this spectrum is deconvoluted (Figure 1c) the glycosylation heterogeneity of the MAB is shown. The average theoretical mass of the G0F/G0F glycoform calculated from the amino acid sequence [2, 42] is $148,057 \mathrm{Da}$. The 

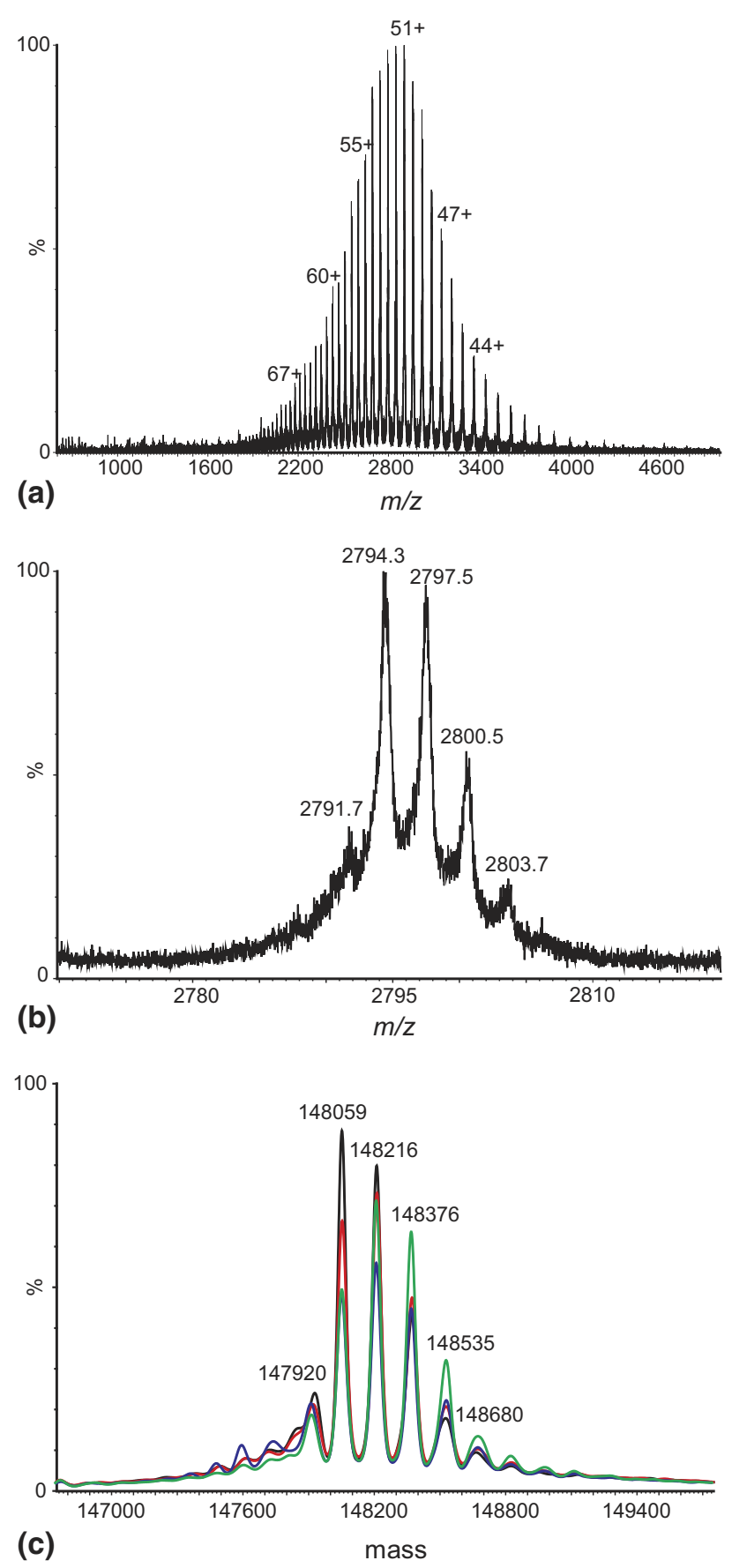

Figure 1. (a) ESI-TOF MS spectra of intact trastuzumab (batch B2033) by LC-MS. Charge state distribution. (b) Zoomed spectrum for the +53 charge state. (c) Deconvoluted mass spectrum showing an overview of the glycosylation heterogeneity and comparing batches B0011 (black), B1142 (green), B2033 (red), and B2045 (purple) by superimposing the spectra.

measured mass of 148,059 (Figure 1c) has a mass difference of $2 \mathrm{Da}$. The theoretical mass calculation does not take into consideration any other possible posttranslational modification, such as C-terminal amide formation from glycine or pyroglutamic acid formation from glutamic acid at the N-terminal since no additional information indicates these post-translation mod- ifications have occurred with the trastuzumab sample. The measured mass on the IMS-Q-TOF instrument represents a mass error of $13 \mathrm{ppm}$ (externally calibrated) and is closer to the theoretical masses compared with the data reported in other papers on different types of mass spectrometers. Le and Bondarenko [43] found a mass of $148,098 \mathrm{Da}$ using an ion trap mass spectrometer, and using a triple quadrupole instrument, we found a mass of $148,066 \mathrm{Da}$ [44] for the same MAB.

Based on the deconvolution masses, peaks observed in Figure 1c can be assigned to known glycoforms of the trastuzumab antibodies: G0/G0F (147920 Da), G0F/ G0F (148059 Da), G0F/G1F (148,216 Da), G0F/G2F or G1F/G1F (148,376 Da), G1F/G2F (148,535 Da), and G2F/G2F (148,680 Da). Additional peaks, which are not explicable with the most common glycan structures, could be artifacts from the deconvolution process. To validate whether these peaks have a connection with the glycan heterogeneity, the sample was reduced to generate subunits which were analyzed in the next step.

Deconvoluted mass spectra for intact trastuzumab for all four batches are normalized and displayed in Figure 1c. The spectra clearly reveal the glycoprofile difference between trastuzumab antibodies from different batches. The observed differences among the batches are experimentally significant since multiple injections of each sample yielded identical results. Figure $1 \mathrm{c}$ reveals that all batches contain equal number of peaks (glycoforms), however, the intensity of each corresponding peak varies greatly among the four batches. The variability is attributed to the microheterogeneity, predominantly galactose heterogeneity, at the conserved N-linked glycosylation site in the Fc region of the antibody. It is well-known that N-linked oligosaccharides can vary during antibody processing.

\section{Analysis of Trastuzumab Subunits}

Reduction of the intact trastuzumab with DTT and subsequent LC/MS allows mass analysis of light chain and heavy chain subunits from a single injection. Figure 2a shows a typical total ion current (TIC) chromatogram of the partially reduced sample desalted with the phenyl desalting column. Two prominent peaks are shown, each representing the light chain and the heavy chain. The light chain is eluted of the column first $(\mathrm{Rt}=11.19$ $\mathrm{min}$ ) followed by the heavy chain (Rt $=13.37 \mathrm{~min}$ ). When the TIC chromatograms of all four batches are superimposed, no differences between batches can be observed. Figures $2 b$ and $d$ show the examples of the summed ESI raw spectra of the light chain and heavy chain from reduced trastuzumab, and the deconvoluted spectra for the light and the heavy chain are also shown in Figures 2c and e.

Deconvoluted spectra for the light chain (Figure 2c) consists of a major peak at 23,438 $\mathrm{Da}$ and two minor peaks at 23,420 and 23,600, respectively. Based on the theoretical mass calculation, the major peak at 23,438 

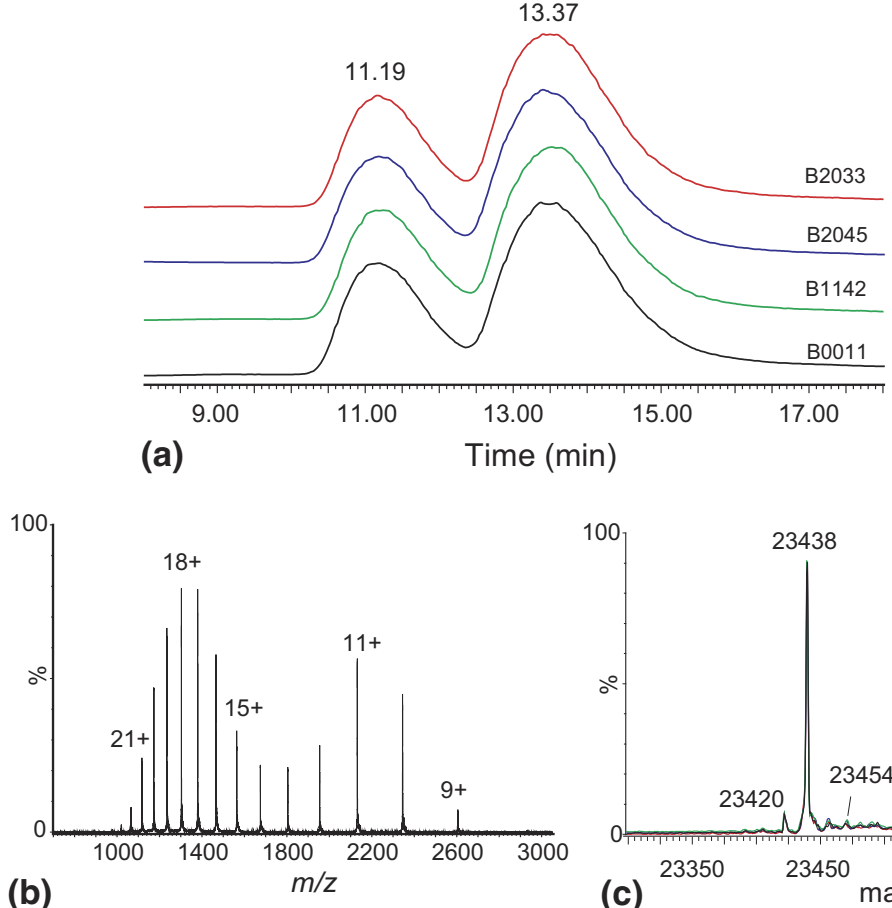

(b)

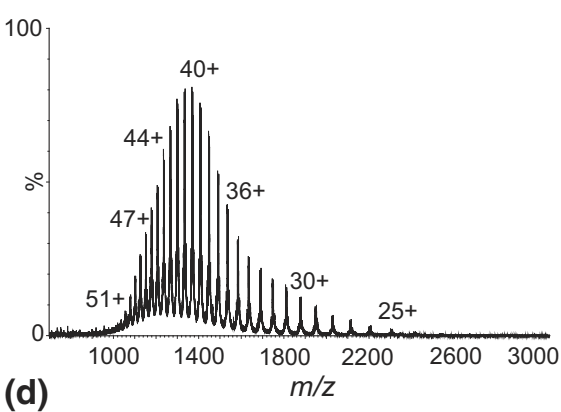

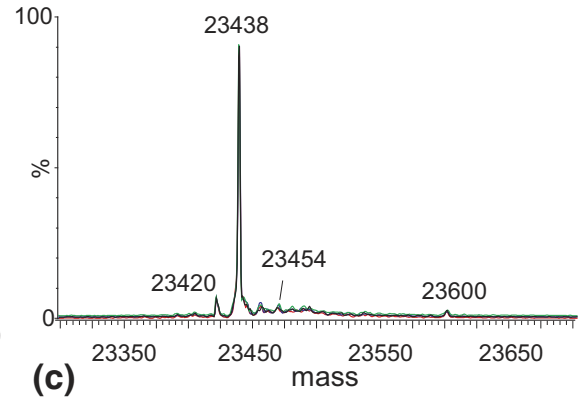

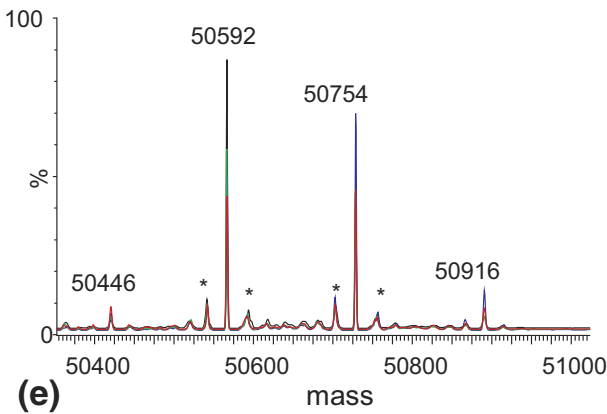

Figure 2. On-line LC/MS analysis of the partially reduced trastuzumab using TOF mode. (a) Total ion chromatogram of the reduced trastuzumab. (b) Combined ESI-TOF spectrum of the light chain $(\mathrm{Rt}=11.2 \mathrm{~min})$. (c) Deconvoluted mass spectrum of the light chain. (d) Combined ESI-TOF raw spectrum of the heavy chain $(\mathrm{Rt}=13.4 \mathrm{~min})$. (e) Deconvoluted mass spectrum of the heavy chain. (a), (c), and (e) compare the different batches B0011 (black), B1142 (green), B2033 (red), and B2045 (purple) by superimposing chromatograms or the spectra, respectively. Asterisks indicate deconvolution artifacts.

Da is assigned to the light chain of trastuzumab. The small peak at 23,420 Da probably results from the loss of water from the light chain molecule. The observed mass difference between the major peak and the minor peak at 23,600 is $162 \mathrm{Da}$ corresponding to a single hexose modification of the light chain. This last structure could not be observed when analyzing trastuzumab at the intact protein level (Figure 1c), demonstrating that the analysis of the reduced MAB has additional value over intact protein analysis. In general, no differences between the different trastuzumab batches can be observed with respect to the light chain of the molecule.

In contrast, significant microheterogeneity was found to reside in the heavy chains. This observation is expected as the presence of oligosaccharides is predominantly located within the Fc region of the molecule. The deconvoluted spectrum of the heavy chain (Figure 2e) shows two major peaks at 50,592 and 50,754 Da and two minor peaks at 50,446 and 50,916 Da. Additionally, there are several extraneous peaks observed in the deconvoluted spectra in Figures $2 \mathrm{c}$ and e (marked with an asterisk). These putative peaks are believed to be deconvolution artifacts as they were not visible on the original ESI mass spectra. Furthermore, the mass and peak intensity of these peaks change when deconvolution parameters (peak widths at half height) were modified. Several deconvolution artifacts and their sources have been reported previously in the literature [23]. The two major peaks in Figure 2e (50,592 and 50,754 Da) have a characteristic mass difference of $162 \mathrm{Da}$, consistent with the heavy chain glycosylations where the core glycan structures from the variants differ from each other by a galactose residue. The minor peak with a mass of 50,916 also has a mass difference of $162 \mathrm{Da}$, correspond- 
ing with a second galactose residue. The minor peak with a mass of 50,445 Da differs 146 Da from the major peak of $50,592 \mathrm{Da}$, implying that the heavy chain contains an oligosaccharide moiety without fucose.

The spectra of the heavy chains from the four batches of trastuzumab are compared. The peak intensities were summed and set to $100 \%$. The amounts of the different glycoforms were calculated as percentage of the total peak intensities. Although the overall glycoform patterns are very similar, it is found that the peak intensity is varied for each of the corresponding glycoform. Table 1 summarizes the relative abundances of G0, G0F, G1F, and G2F forms for all four production batches of trastuzumab. The relative quantification of the glycoforms was calculated based on the peak intensities of the deconvoluted mass spectra of the heavy chain (Figure 2e). These values agree well with the results obtained from 2-aminobenzamide labeled released glycans from the same batch sample (see Supplemental Materials for batch B2045, which can be found in the electronic version of this article). To check the reproducibility of the method, batch B0011 and B2033 were reduced and analyzed on five different days with multiple injections. The results show that the analysis is reproducible within the day and also between days. The relative standard deviation (RSD) for peak intensity is generally about 5\% with exception of the glycoform G0, which shows a relatively larger variation $(\sim 20 \%)$. This large variation is attributed to the low peak intensity observed for the G0 form. It is the least intensive peak among all the glycoforms. As the RSD values are very low, the results in Table 1 clearly show that there are variations in glycosylation profile between the batches. In batches B0011 and B1142 the G0F glycan is the most prominent form, however in batch B2045, the G1F glycoform is the most prominent one. For Batch B2033, the intensities between glycoform G0F and G1F are approximately equal. Batch B2033 shows the highest G0 percentage compared with the other three batches, and batch B2045 shows more G2F glycoform exists to this preparation compared with the other three batches.

These results demonstrate the application of the described method for the lot-to-lot comparison of the glycosylation profile of a therapeutic monoclonal antibody. The mentioned glycoforms, however, are only determined by deconvoluting the spectrum of intact and

Table 1. Comparison of the four trastuzumab batches for the relative abundance of G0, G0F, G1F, and G2F glycans measured on the deconvoluted ESI mass spectrum of the heavy chain (Figure 2)

\begin{tabular}{lcccc}
\hline & \multicolumn{4}{c}{ Glycoforms of heavy chain (\%) } \\
\cline { 2 - 5 } Batch & G0 & G0F & G1F & G2F \\
\hline \hline B0011 & 4.96 & 52.8 & 35.8 & 6.47 \\
B2033 & 9.59 & 39.8 & 40.0 & 10.6 \\
B1142 & 6.53 & 47.4 & 38.4 & 7.65 \\
B2045 & 6.12 & 35.9 & 44.7 & 13.3 \\
\hline
\end{tabular}

reduced trastuzumab and by searching for the most common glycoforms. No real structural elucidation was performed. For the structural characterization of the glycoforms of trastuzumab the reduced molecule was made smaller again and was subject to a trypsin digestion.

\section{Characterization of Glycopeptides}

To further elucidate the glycan sequencing and to determine the glycosylation site for the glycosylation observed at the intact or the subunit level, the method developed by Olivova et al. [37] was adapted in this work. The method essentially includes two sequential steps to characterize glycopeptides from trastuzumab digestion without any glycopeptide enrichment. In the first step, the LC elution time and the precursor mass of the glycopeptides are determined by a multiplexed parallel fragmentation method. In the second step, the glycan sequence, as well as the glycopeptide backbone sequence, is determined for all the glycopeptides revealed in the first step.

Among all the tryptic peptides of trastuzumab, the overwhelming majority of the tryptic peptides are not glycosylated. Since there is no prior enrichment involved, the first step in the analysis was to identify the peptide that is glycosylated as well as their elution time. Under ESI conditions, mass spectral detection of glycopeptides during on-line LC/MS analysis is normally achieved by precursor ion scanning or cone fragmentation for reporter oxonium fragment ions (e.g., $\mathrm{m} / \mathrm{z}$ 204.087) derived from glycan fragments [45, 46]. To obtain high specificity of precursor-ion scanning, it is pivotal to use high-resolution, high accuracy fragment ion selection on a hybrid quadrupole time-of-flight (Q-TOF) mass spectrometer [37, 47].

The multiplexed parallel fragmentation method takes advantage of the high mass accuracy that a Q-TOF mass spectrometer offers in precursor ion scanning. The precursor masses of peptides eluting off the RP column were obtained in a low-energy scan in which peptides were not fragmented. In the following scan, the collision energy was increased to a level such that only the fragmentation of glycan moiety of the glycopeptide was induced. It is known that glycans are labile, and less collision energy is required to induce the cleavage of the glycosidic bonds compared with that of the peptide backbone. This step of fragmentation produced oxonium ions while minimizing peptide backbone cleavage. The appearance of oxonium ions marked the elution time of glycopeptides. The intact mass of the glycopeptides were acquired from the low-energy scan. Because there was no precursor selection at the elevated energy stage, all the eluting peptides were subjected to the same collision condition. However, only glycopeptides produced glycan reporter ions. This data acquisition procedure was repeated across the whole LC gradient.

Figure 3 shows LC/MS chromatograms from the analysis of a tryptic digest of trastuzumab (batch B0011) 

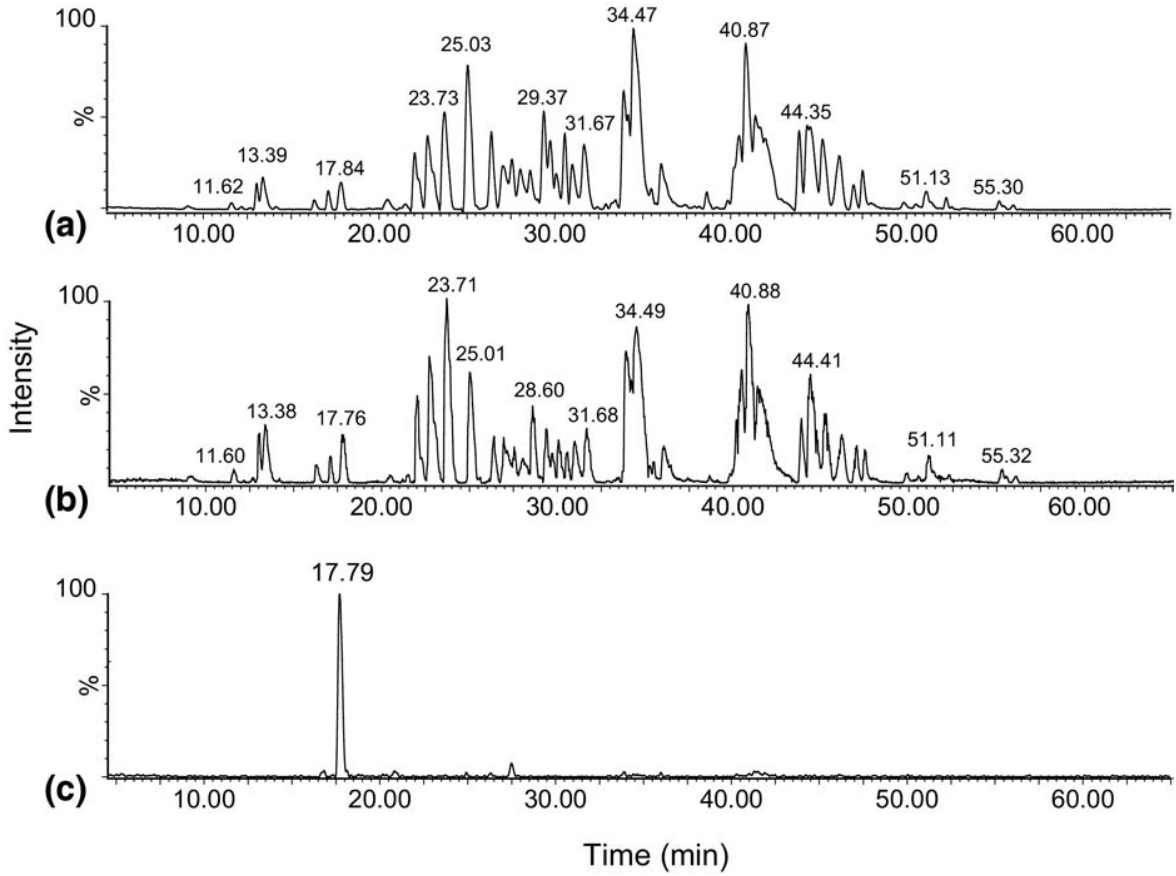

Figure 3. LC/MS/MS ${ }^{\mathrm{E}}$ analysis of a tryptic digest of trastuzumab (batch B0011). The base peak intensity chromatograms for the tryptic precursors and associated fragment ions are provided in each of the two acquisition channels: (a) LC-MS, Low-energy; (b) LC/MS ${ }^{\mathrm{E}}$, elevated energy; (c) reconstructed ion chromatogram for oxonium ions $(\mathrm{m} / \mathrm{z} 204.084$ and 366.139) from the elevated energy data.

using the multiplexed parallel fragmentation approach. Figure 3a depicts the MS responses of tryptic peptides at low collision energy during the LC gradient run. Figure $3 b$ shows the responses of the peptides at the elevated energy, and Figure $3 c$ displays the reconstructed ion chromatogram for oxonium ions $(\mathrm{m} / \mathrm{z}$ 204.084 and 366.139) from the elevated energy data. The dominant peak at retention time of 17.79 min is attributed to the co-eluting (Figure $3 \mathrm{c}$ ) glycopeptides. To identify all the precursors responsible for the generation of oxonium ions, scans spanning the elution time of oxonium ions are summed for both low- and highenergy data and presented in Figures $4 a$ and b, respectively. Comparison of Figure $4 a$ and $b$ reveals that the signal intensities of several peaks in the $\mathrm{m} / \mathrm{z}$ range between 800 and 1000 decreased in the elevated-energy spectrum. The mass difference of the four peaks (labeled by $\left.{ }^{*}\right)$, which are triply charged $(m / z$ 829.96, 878.66, $932.68,986.70)$, matches the glycoform patterns observed in the intact and reduced trastuzumab analyses, suggesting the four glycopeptides contain the same peptide backbone and represent the G0, G0F, G1F, G2F glycoforms of the glycopeptides. These four different masses were selected as the precursors for the second step of the experiment.

Peaks corresponding to the doubly charged species of the four glycopeptides are also observed in both low and high-energy scans ( $m / z 1244.52$ 1317.49, 1398.55, 1479.58 , and all labeled by [filled square]). Each of these peaks shows similar ion intensity in the elevated energy data compared with the low-energy data, suggesting little fragmentation taking place for the doubly charged glycopeptides under the relatively low collision energy setting. It is known that less energy is needed to fragment the same species with higher charge states. The other peaks observed in the spectra (e.g., $m / z$ : $1134.92,1215.97)$ come from glycopeptides with truncated glycan moieties in comparison with the G0, G0F, G1F, G2F glycoforms. These species were generated from the fragmentation of the glycopeptides precursor ions.

Although only ions with $3+$ charge states were fragmented predominantly in the multiplexed parallel fragmentation approach, the data clearly shows good sensitivity and selectivity in identifying glycopeptides for further characterizations. The MS sensitivity is ascribed to that fact the co-eluting glycopeptides all contributed to the overall signal intensity of oxonium ions. The selectivity comes from the collision energy settings, which are below the typical energy used for the fragmentation of the peptide backbone, and the formation of potentially interfering fragment ions is considerably reduced. As a result, the formation of the reporter fragment ions is purely generated by glycan fragmentation. The low collision energy settings employed were distinct from most other types of precursor ion scanning methods used for the identification of protein modifications that require higher collision energies to generate the reporter fragment ion of interest.

The determination of the molecular masses and retention times for these glycopeptides allow us to focus only on the glycopeptides of interest for the 

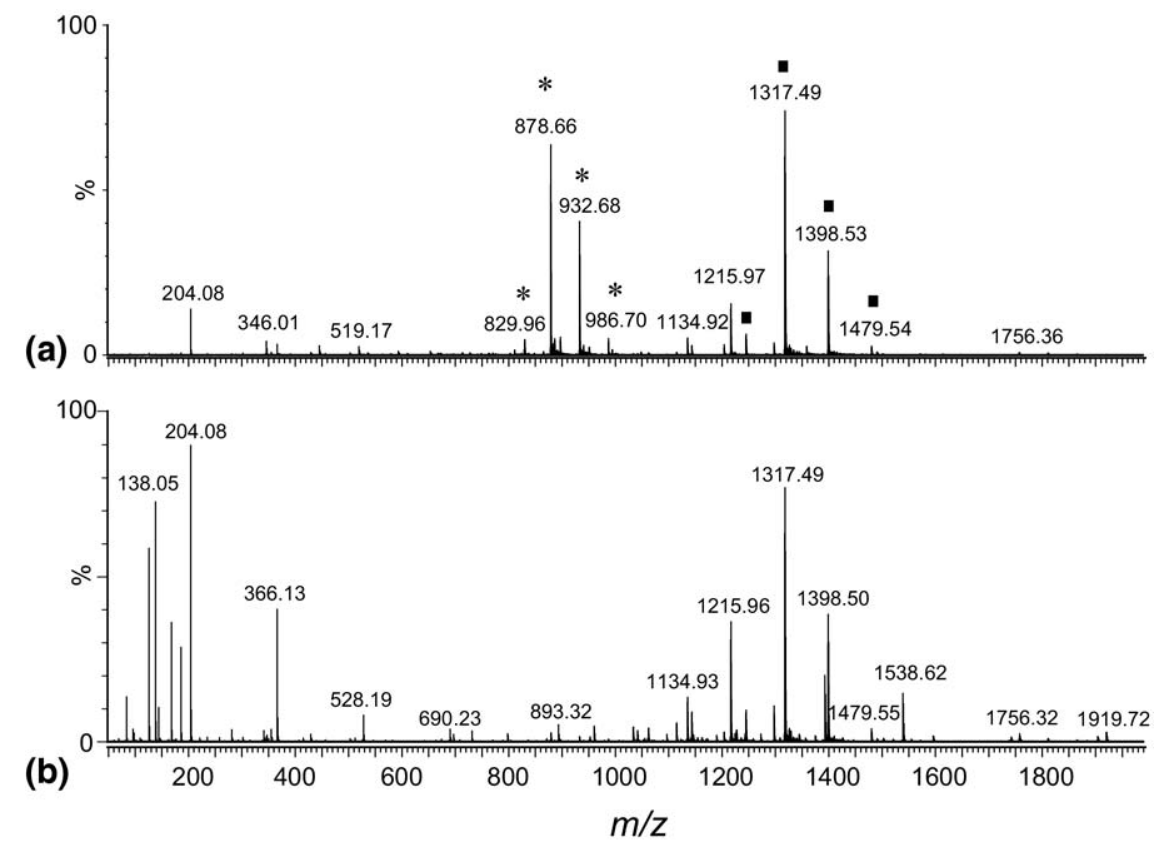

Figure 4. ESI mass spectra of glycopeptides from LC/MS/MS ${ }^{\mathrm{E}}$ analysis. (a) Combined precursor mass spectrum for the retention time at $17.8 \mathrm{~min}$ from the low-energy scan. (b) Combined fragment ion mass spectrum for the retention time at $17.8 \mathrm{~min}$ from the elevated energy scan.

verification of the glycan sequence and glycosylation site. Each of the four protonated glycopeptides was sequentially selected by the quadrupole ion filter and subsequently fragmented in the TRAP cell with energy sufficient to induce extensive glycan fragmentation while peptide backbone cleavage was kept at minimum. The fragment ions were then transported into the ion-mobility cell where ions were separated into individual ion packages on the basis of their ion mobility (combination of size, shape, and charge as well as mass). A two-dimensional plot of ion drift time versus the $\mathrm{m} / \mathrm{z}$ values from a glycopeptide $(\mathrm{m} / \mathrm{z}$ 932.68), which is fragmented in the TRAP cell, followed by ion mobility separation is shown in Figure 5a. The ion-mobility separated fragment ions were subsequently transported through the TRANSFER T-wave collision cell in which the collision energy was alternated between two settings (low and high). When the collision energy of the TRANSFER cell is set at a low-energy (normally at $5 \mathrm{~V}$ ), no additional fragmentation takes place in the TRANSFER T-wave. Fragment ions observed in this scan would only represent the fragments generated in the TRAP T-wave collision cell. Thus MS/MS data recorded in this process would contain information only for the deduction of the glycan sequence of the glycopeptides. Figure $5 b$ shows a summed MS/MS spectrum from a glycopeptide $(\mathrm{m} / \mathrm{z}$ 932.68) fragmented in the TRAP cell as a representative example for all the glycopeptides investigated. Multiply charged ions in the raw data were transformed to singly charged ones using MaxEnt3. From Figure 5b, it can be seen that fragmentation of the peptide backbone under the chosen conditions is negligible. The MS/MS spectrum exclusively exhibits the cleavage of glycosidic linkages, thus revealing information on the glycan moiety. On the basis of the fragment ion analysis, the general structure of an N-linked complex type biantennary glycan could be deduced and confirmed [10].

Mass analysis on the fragment ions generated in the TRAP T-wave collision cell shows the existence of a fragment ion with a $\mathrm{m} / \mathrm{z}$ value of 1392.57 . This ion comes from a peptide that contains a single HexNAc attached to the glycosylated residue of the $\mathrm{N}$-glycopeptide. To obtain the fragmentation of this precursor, the collision energy of the TRANSFER T-wave was increased to a higher energy (typically 80 to $120 \mathrm{~V}$ ) in the second scan, and the ion-mobility separated product ions are subjected to a second-generation fragmentation at which peptide backbone cleavage would occur. Figure 6a shows the corresponding data (in DriftScope software, Waters Corp., Milford, MA, USA) obtained from the $\mathrm{N}$-glycopeptide fragments after the collision energy of the TRANSFER T-wave was raised up to 100 $\mathrm{V}$. Figure $6 \mathrm{~b}$ displays the mass spectrum that is generated by combining all the fragmentation data from drift time 7.2 to $8.3 \mathrm{~ms}$. Since these product ions all share the same drift time as the precursor ion $(\mathrm{m} / \mathrm{z} 1392.57$, see Figure 5a), these fragment ions all come from the same precursor, and the reconstructed spectrum over that range of drift times corresponds to the peptide sequence of the precursor. From Figure $6 b$, the peptide sequence (EEQYNSTYR) can be easily confirmed, and the glycan attachment site is inferred.

The approach to fragment mobility-separated product ions using the instrument is rather similar to an $\mathrm{MS}^{3}$ type experiment performed on an ion-trap mass spec- 


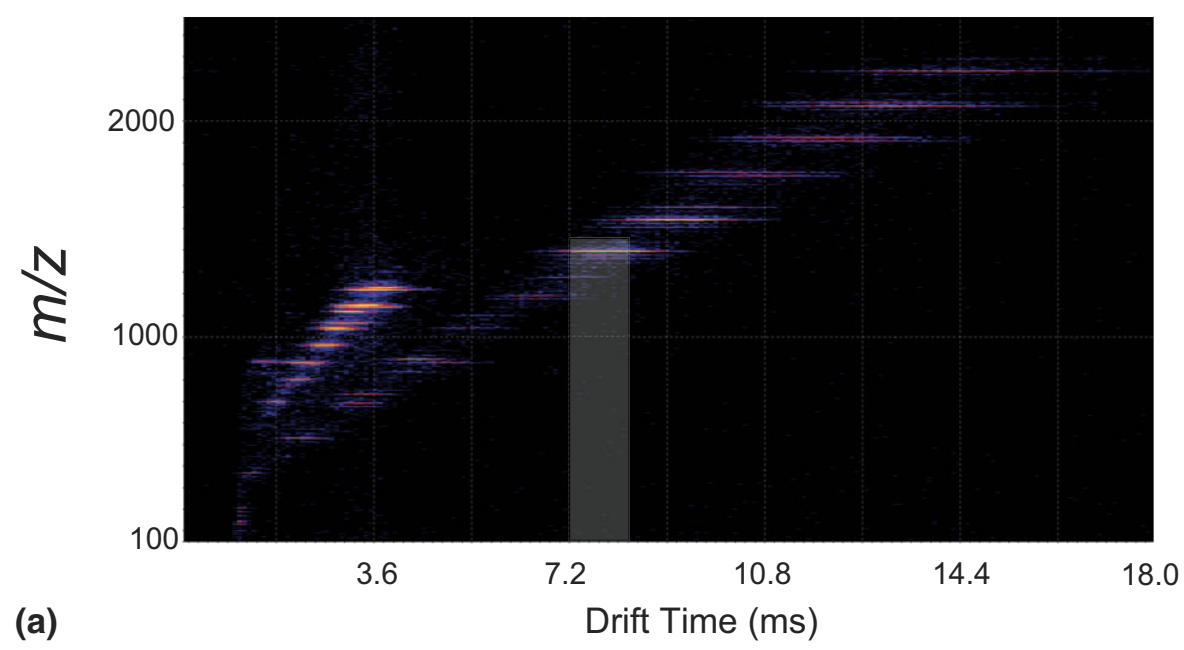

(a)

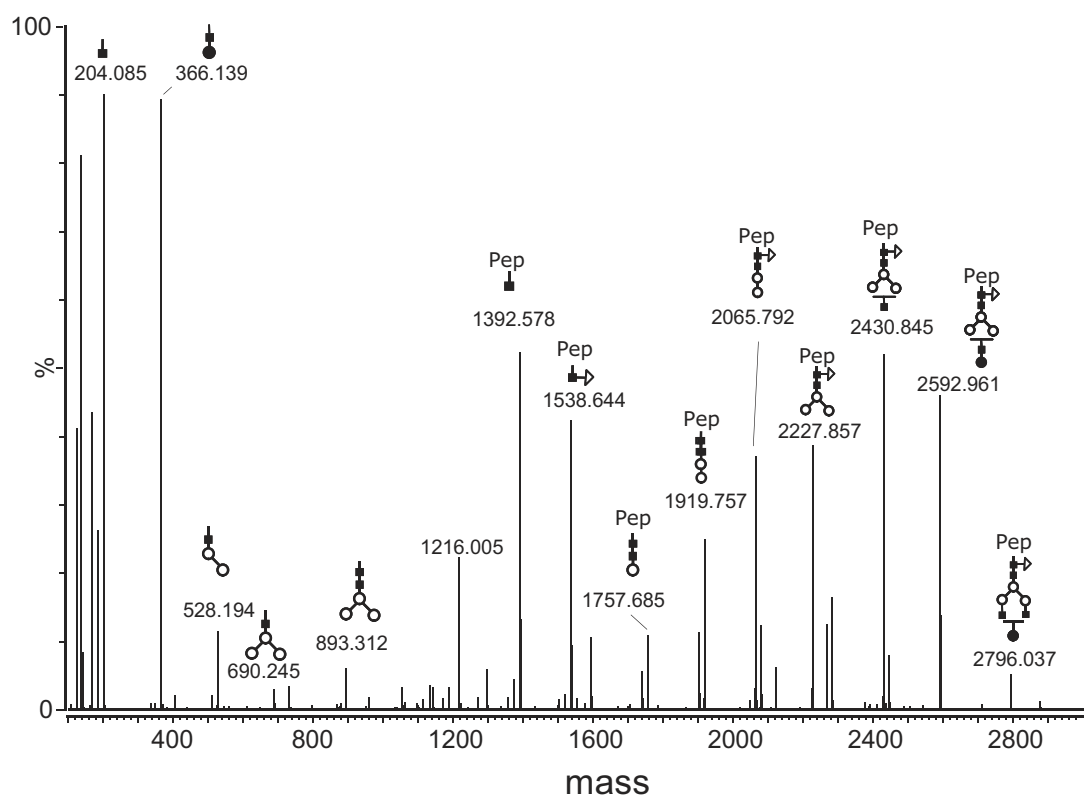

Figure 5. Glycopeptides analysis using LC/ESI-Q-IMS-TOF MS. (a) Two-dimensional plot of ion drift time versus the $m / z$ values for the $[\mathrm{M}+3 \mathrm{H}]^{3+}$ ion at $m / z$ 932.70, which was subject to fragmentation in the TRAP cell, followed by ion-mobility separation. The highlighted region shows the drift-time span for the fragment ion with $\mathrm{m} / \mathrm{z}$ 1392.57. (b) MS/MS spectrum for the parent $[\mathrm{M}+$ $3 \mathrm{H}]^{3+}$ ion at $\mathrm{m} / \mathrm{z} 932.70$ from Figure 4 . The fragment ions are generated by the CID process in the TRAP cell to yield information on the glycan sequence.

trometer. However, the method has the advantage that all first- and second-generation product ions are recorded in parallel. In addition, the two-step sequential fragmentation process allows a user to optimize the collision energy for glycosidic and peptide fragmentation separately to maximize the information obtained in each step by avoiding the loss of glycan sequence information due to the higher energy applied for peptide backbone fragmentation. The ability to reduce collision energy applied specifically for glycosidic fragmentation minimizes the peptide backbone fragment ions that can complicate the interpretation of glycan sequences. Because the process de-couples the glycan fragmentation process from peptide backbone fragmentation, it is conceivable that this approach can be used for different sizes/types of glycopeptides including O-linked glycopeptides.

The elucidated glycan structures matched the G0, G0F, G1F, and G2F forms reported in literature [48]. Although no novel glycan structure is discovered, the ability to rapidly obtain glycoprofile of a therapeutic monoclonal antibody and subsequently confirm the glycan structures is very much needed in the biopharmaceutical industry. Blocking HER2 by trastuzumab is mediated by the antigen-binding site of the $\mathrm{mAb}$ and may contribute to some or all of the observed clinical efficacy of these agents. However, clinical efficacy may also be a result of, at least in some part, antibodyspecific mechanisms mediated through the Fc domain of the mAb [49]. Recently, Karagiannis et al. [50] inves- 

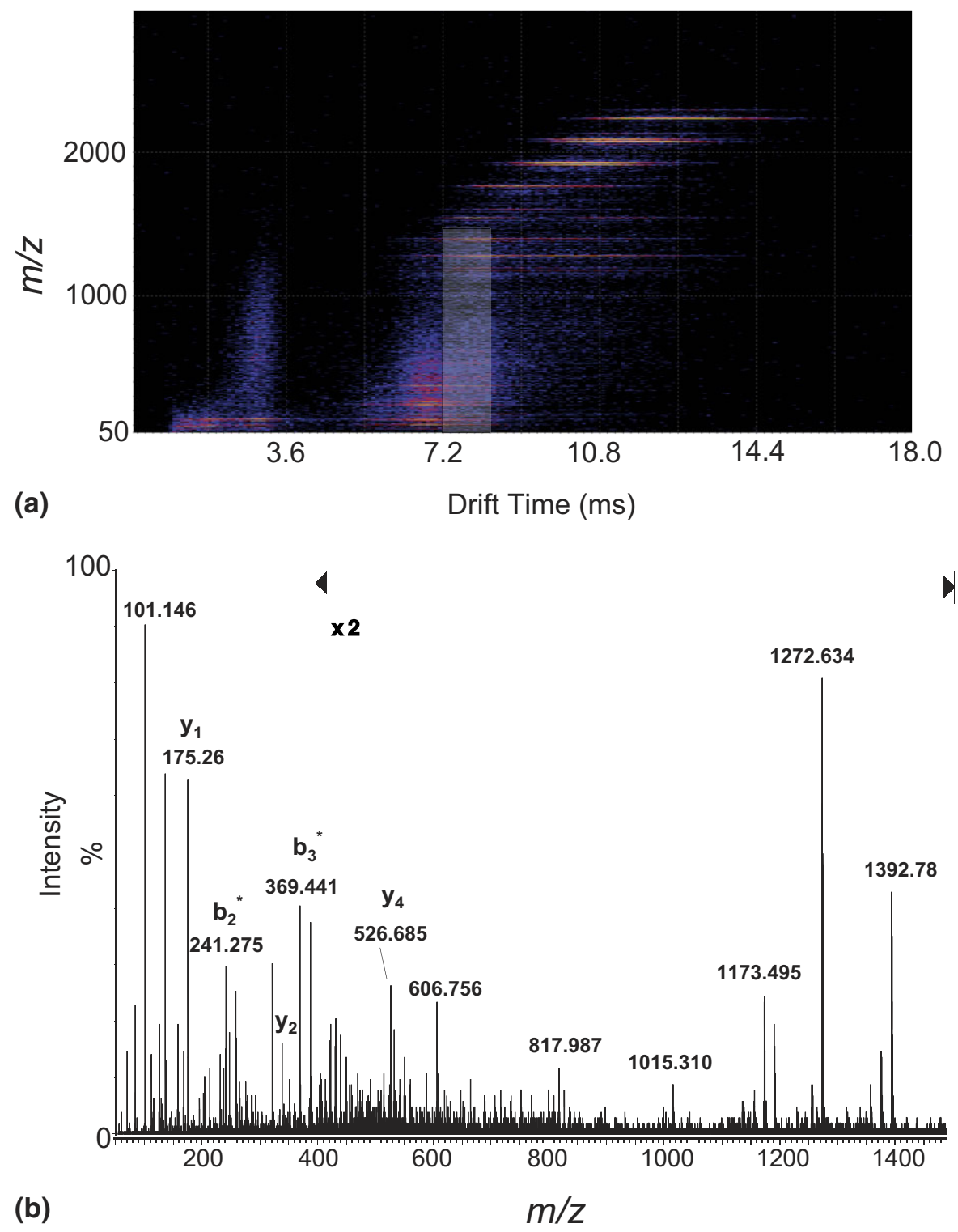

Figure 6. Obtaining the N-glycosylation site by performing second stage fragmentation on the product ions generated in the TRAP T-wave. (a) Two-dimensional plot $(\mathrm{m} / \mathrm{z}$ versus drift time) showing the second generation of fragment ions following CID in the TRANSFER T-wave (CE of $100 \mathrm{~V})$. The boxed highlight region represents the distribution of the second generation product ions from the precursor $\mathrm{m} / \mathrm{z} 1392.58$ as shown in DriftScope (note the precursor and fragments share the same drift time). (b) Reconstructed MS spectrum from the boxed region in Figure 6A with sequence ions annotated.

tigated that trastuzumab functions in antibody-dependent cell-mediated phagocytosis (ADCP). The presence of 'core' oligosaccharide is essential for the expression of IgG-Fc effector functions, and the addition of galactose residues has a variable influence on the efficacy of specific functions [5]. The presence or absence of galactose on IgG glycans correlates in some but not all monoclonal IgG antibodies with respect to the binding to the Fc receptor [48]. The impact of the presence of galactose and fucose residues on the binding of trastuzumab to Fc receptors has not been reported. However, it is striking that we found a difference in galactose residues between different production lots for a $\mathrm{mAb}$, which is already registered.
Therefore, further study is required to demonstrate if there is any correlation between the drug efficacy and the lot-to-lot differences in glycoprofile. This work, however, provides an example of how the ESI-Q-IMTOF platform can be used for lot-to-lot consistency testing and structural elucidation of glycans when new MABs are developed.

\section{Conclusions}

In this work, we have presented a rapid method to distinguish the lot-to-lot heterogeneity in glycosylation profile of the commercially available monoclonal antibody trastuzumab. The batches were first compared at 
the intact level, showing differences between the four production batches tested. A subsequent analysis of the reduced antibody allowed for additional confirmation of the glycoprofile difference. In addition, by using the unique ion-mobility functionality of the instrument, the structures of the glycan moieties are confirmed in a simple experiment. The results demonstrate the current analytical method is very effective for monitoring and validating the glycan variations of production lots during the process of antibody development. The FDA and other regulatory agencies may require more data on the analytical characterization of MABs in the future and this platform is suitable to fulfill these needs. Lot-to-lot consistency testing is important because molecular heterogeneity of MABs may affect stability and potency.

\section{Appendix A Supplementary Material}

Supplementary material associated with this article may be found in the online version at doi:10.1016/ j.jasms.2009.07.017.

\section{References}

1. Brekke, O. H.; Sandlie, I. Therapeutic Antibodies for Human Diseases at the Dawn of the Twenty-First Century. Nat. Rev. Drug Discov. 2003, 2 , 52-62.

2. Carter, P.; Presta, L.; Gorman, C. M.; Ridgway, J. B.; Henner, D.; Wong, W. L.; Rowland, A. M.; Kotts, C.; Carver, M. E.; Shepard, H. M. Humanization of an Anti-p185HER2 Antibody for Human Cancer Therapy. Proc. Natl. Acad. Sci. U.S.A. 1992, 89, 4285-4289.

3. Hudis, C. A. Trastuzumab-Mechanism of Action and Use in Clinical Practice. N. Engl. J. Med. 2007, 357, 39-51.

4. Jefferis, R. Glycosylation of Recombinant Antibody Therapeutics. Biotechnol. Prog. 2005, 21, 11-16.

5. Walsh, G.; Jefferis, R. Post-Translational Modifications in the Context of Therapeutic Proteins. Nat. Biotechnol. 2006, 24, 1241-1252.

6. Masuda, K.; Yamaguchi, Y.; Kato, K.; Takahashi, N.; Shimada, I.; Arata, Y. Pairing of Oligosaccharides in the Fc Region of Immunoglobulin G. FEBS Lett. 2000, 473, 349-357.

7. Krapp, S.; Mimura, Y.; Jefferis, R.; Huber, R.; Sondermann, P. Structural Analysis of Human IgG-Fc Glycoforms Reveals a Correlation Between Glycosylation and Structural Integrity. J. Mol. Biol. 2003, 325, 979-989.

8. Lim, A.; Reed-Bogan, A.; Harmon, B. J. Glycosylation Profiling of a Therapeutic Recombinant Monoclonal Antibody with Two N-Linked Glycosylation Sites Using Liquid Chromatography Coupled to a Hybrid Quadrupole Time-of-Flight Mass Spectrometer. Anal. Biochem. 2008, 375, 163-172.

9. U.S. Food and Drug Administration: Center for Drug Evaluation and Research; Center for Biologics Evaluation and Research. Guidance for Industry. Q6B Specifications: Test Procedures and Acceptance Criteria for Biotechnological/Biological Products. Available: www. fda. gov/ cder/guidance/Q6Bfnl. PDF 1999.

10. Rothman, R. J.; Warren, L.; Vliegenthart, J. F.; Hard, K. J. Clonal Analysis of the Glycosylation of Immunoglobulin G Secreted by Murine Hybridomas. Biochemistry. 1989, 28, 1377-1384.

11. Grebenau, R. C.; Goldenberg, D. M.; Chang, C. H.; Koch, G. A.; Gold, D. V.; Kunz, A.; Hansen, H. J. Microheterogeneity of a Purified IgG1 Due to Asymmetric Fab Glycosylation. Mol. Immunol. 1992, 29, 751-758.

12. Kunkel, J. P.; Jan, D. C.; Butler, M.; Jamieson, J. C. Comparisons of the Glycosylation of a Monoclonal Antibody Produced Under Nominally Identical Cell Culture Conditions in Two Different Bioreactors. Biotechnol. Prog. 2000, 16, 462-470.

13. Patel, T. P.; Parekh, R. B.; Moellering, B. J.; Prior, C. P. Different Culture Methods Lead to Differences in Glycosylation of a Murine IgG Monoclonal Antibody. Biochem. J. 1992, 285(Pt3), 839-845.

14. Bailey, M. J.; Hooker, A. D.; Adams, C. S.; Zhang, S.; James, D. C. A Platform for High-Throughput Molecular Characterization of Recombinant Monoclonal Antibodies. J. Chromatogr. B Analyt. Technol. Biomed. Life Sci. 2005, 826, 177-187.

15. Hills, A. E.; Patel, A.; Boyd, P.; James, D. C. Metabolic Control of Recombinant Monoclonal Antibody N-Glycosylation in GS-NS0 Cells. Biotechnol. Bioeng. 2001, 75, 239-251.

16. Lyubarskaya, Y.; Houde, D.; Woodard, J.; Murphy, D.; Mhatre, R. Analysis of Recombinant Monoclonal Antibody Isoforms by Electros- pray Ionization Mass Spectrometry as a Strategy for Streamlining Characterization of Recombinant Monoclonal Antibody Charge Heterogeneity. Anal. Biochem. 2006, 348, 24-39.

17. Kamoda, S.; Kakehi, K. Evaluation of Glycosylation for Quality Assurance of Antibody Pharmaceuticals by Capillary Electrophoresis. Electrophoresis. 2008, 29, 3595-3604.

18. Ma, S.; Nashabeh, W. Carbohydrate Analysis of a Chimeric Recombinant Monoclonal Antibody by Capillary Electrophoresis with LaserInduced Fluorescence Detection. Anal. Chem. 1999, 71, 5185-5192.

19. Serrato, J. A.; Hernandez, V.; Estrada-Mondaca, S.; Palomares, L.A.; Ramirez, O. T. Differences in the Glycosylation Profile of a Monoclonal Antibody Produced by Hybridomas Cultured in Serum-Supplemented, Serum-Free or Chemically Defined Media. Biotechnol. Appl. Biochem. 2007, 47, 113-124.

20. Adamczyk, M.; Gebler, J. C.; Harrington, C. A.; Sequeira A. F. The Use of Electrospray Ionization Mass Spectrometry to Distinguish the Lotto-Lot Heterogeneity of an Antigen Specific Monoclonal Antibody from a Specific Cellular Clone. Eur. Mass Spectrom. 1999, 5, 165-168.

21. Wan, H. Z.; Kaneshiro, S.; Frenz, J.; Cacia, J. Rapid Method for Monitoring Galactosylation Levels During Recombinant Antibody Production by Electrospray Mass Spectrometry with Selective-Ion Monitoring. J. Chromatogr. A 2001, 913, 437-446.

22. Siemiatkoski, J.; Lyubarskaya, Y.; Houde, D.; Tep, S.; Mhatre, R. A Comparison of Three Techniques for Quantitative Carbohydrate Analysis Used in Characterization of Therapeutic Antibodies. Carbohydr. Res. 2006, 341, 410-419.

23. Gadgil, H. S.; Pipes, G. D.; Dillon, T. M.; Treuheit, M. J.; Bondarenko, P. V. Improving Mass Accuracy of High Performance Liquid Chromatography/Electrospray Ionization Time-of-Flight Mass Spectrometry of Intact Antibodies. J. Am. Soc. Mass Spectrom. 2006, 17, 867-872.

24. Huang, L.; Biolsi, S.; Bales, K. R.; Kuchibhotla, U. Impact of Variable Domain Glycosylation on Antibody Clearance: an LC/MS Characterization. Anal. Biochem. 2006, 349, 197-207.

25. Mimura, Y.; Ashton, P. R.; Takahashi, N.; Harvey, D. J.; Jefferis, R. Contrasting Glycosylation Profiles Between Fab and Fc of a Human IgG Protein Studied by Electrospray Ionization Mass Spectrometry. J. Immunol. Methods 2007, 326, 116-126.

26. Gadgil, H. S.; Bondarenko, P. V.; Pipes, G.; Rehder, D.; McAuley, A.; Perico, N.; Dillon, T.; Ricci, M.; Treuheit, M. The LC/MS Analysis of Glycation of IgG Molecules in Sucrose Containing Formulations. J. Pharm. Sci. 2007, 96, 2607-2621.

27. Beck, A.; Bussat, M. C.; Zorn, N.; Robillard, V.; Klinguer-Hamour, C. Chenu, S.; Goetsch, L.; Corvaia, N.; Van Dorsselaer, A.; Haeuw, J. F. Characterization by Liquid Chromatography Combined with Mass Spectrometry of Monoclonal Anti-IGF-1 Receptor Antibodies Produced in CHO and NS0 Cells. J. Chromatogr. B Analyt. Technol. Biomed. Life Sci. 2005, 819, 203-218.

28. Kroon, D. J.; Freedy, J.; Burinsky, D. J.; Sharma, B. Rapid Profiling of Carbohydrate Glycoforms in Monoclonal Antibodies Using MALDI/ TOF Mass Spectrometry. J. Pharm. Biomed. Anal. 1995, 13, 1049-1054.

29. Harvey, D. J. Matrix-Assisted Laser Desorption/Ionization Mass Spectrometry of Carbohydrates and Glycoconjugates. Int. J. Mass Spectrom. 2003, 226, 1-35.

30. Chen, X.; Flynn, G. C. Analysis of N-Glycans from Recombinant Immunoglobulin $\mathrm{G}$ by On-Line Reversed-Phase High-Performance Liquid Chromatography/Mass Spectrometry. Anal. Biochem. 2007, 370, 147-161.

31. Rehder, D. S.; Dillon, T. M.; Pipes, G. D.; Bondarenko, P. V. ReversedPhase Liquid Chromatography/Mass Spectrometry Analysis of Reduced Monoclonal Antibodies in Pharmaceutics. J. Chromatogr. A 2006, $1102,164-175$

32. Wang, F.; Nakouzi, A.; Angeletti, R. H.; Casadevall, A. Site-Specific Characterization of the N-Linked Oligosaccharides of a Murine Immunoglobulin M by High-Performance Liquid Chromatography/Electrospray Mass Spectrometry. Anal. Biochem. 2003, 314, 266-280.

33. Sheeley, D. M.; Merrill, B. M.; Taylor, L. C. Characterization of Monoclonal Antibody Glycosylation: Comparison of Expression Systems and Identification of Terminal Alpha-Linked Galactose. Anal. Biochem. 1997, 247, 102-110.

34. Wang, Y.; Wu, S. L.; Hancock, W. S. Approaches to the Study of N-Linked Glycoproteins in Human Plasma Using Lectin Affinity Chromatography and Nano-HPLC Coupled to Electrospray Linear Ion Trap-Fourier Transform Mass Spectrometry. Glycobiology 2006, 16, $514-$ 523.

35. Sinha, S.; Pipes, G.; Topp, E. M.; Bondarenko, P. V.; Treuheit, M. J. Gadgil, H. S. Comparison of LC and LC/MS Methods for Quantifying N-Glycosylation in Recombinant IgGs. J. Am. Soc. Mass Spectrom. 2008, 19, 1643-1654.

36. Yu, L.; Remmele, R. L., Jr.; He, B. Identification of N-Terminal Modification for Recombinant Monoclonal Antibody Light Chain Using Partial Reduction and Quadrupole Time-of-Flight Mass Spectrometry. Rapid Commun. Mass Spectrom. 2006, 20, 3674-3680.

37. Olivova, P.; Chen, W.; Chakraborty, A. B.; Gebler, J. C. Determination of N-Glycosylation Sites and Site Heterogeneity in a Monoclonal Antibody by Electrospray Quadrupole Ion-Mobility Time-of-Flight Mass Spectrometry. Rapid Commun. Mass Spectrom. 2008, 22, 29-40.

38. Yu, Y. Q.; Gilar, M.; Kaska, J.; Gebler, J. C. A Rapid Sample Preparation Method For Mass Spectrometric Characterization of N-Linked Glycans Rapid Commun. Mass Spectrom. 2005, 19, 2331-2336. 
39. Pringle, S. D.; Giles, K.; Wildgoose, J. L.; Williams, J. P.; Slade, S. E.; Thalassinos, K.; Bateman, R. H.; Bowens, M. T.; Scrivens, J. H. An Investigation of the Mobility Separation of Some Peptide and Protein Ions Using a New Hybrid Quadrupole/Traveling Wave IMS/oa-TOF Instrument. Int. J Mass Spectrom. 2007, 261, 1-12.

40. Giles, K.; Pringle, S. D.; Worthington, K. R.; Little, D.; Wildgoose, J. L.; Bateman, R. H. Applications of a Traveling Wave-Based Radio-FrequencyOnly Stacked Ring Ion Guide. Rapid Commun. Mass Spectrom. 2004, 18, 2401-2414.

41. Silva, J. C.; Denny, R.; Dorschel, C. A.; Gorenstein, M.; Kass, I. J.; Li, G. Z.; McKenna, T.; Nold, M. J.; Richardson, K.; Young, P.; Geromanos, S. Quantitative Proteomic Analysis by Accurate Mass Retention Time Pairs. Anal. Chem. 2005, 77, 2187-2200.

42. Harris, R. J.; Kabakoff, B.; Macchi, F. D.; Shen, F. J.; Kwong, M.; Andya, J. D.; Shire, S. J.; Bjork, N.; Totpal, K.; Chen, A. B. Identification of Multiple Sources of Charge Heterogeneity in a Recombinant Antibody. J. Chromatogr. B Biomed. Sci. Appl. 2001, 752, 233-245.

43. Le, J. C.; Bondarenko, P. V. Trap for MAbs: Characterization of Intact Monoclonal Antibodies Using Reversed-Phase HPLC On-Line with Ion-Trap Mass Spectrometry. J. Am. Soc. Mass Spectrom. 2005, 16, 307-311

44. Damen, C. W.; Rosing, H.; Schellens, J. H.; Beijnen, J. H. Quantitative Aspects of the Analysis of the Monoclonal Antibody Trastuzumab Using High-Performance Liquid Chromatography Coupled with Electrospray Mass Spectrometry. J. Pharm. Biomed. Anal. 2008, 46, 449-455.
45. Carr, S. A.; Huddleston, M. J.; Bean, M. F. Selective Identification and Differentiation of N- and O-Linked Oligosaccharides in Glycoproteins by Liquid Chromatography-Mass Spectrometry. Protein Sci. 1993, 2 183-196.

46. Huddleston, M. J.; Bean, M. F.; Carr, S. A. Collisional Fragmentation of Glycopeptides by Electrospray Ionization LC/MS and LC/MS/MS: Methods for Selective Detection of Glycopeptides in Protein Digests. Anal. Chem. 1993, 65, 877-884.

47. Jebanathirajah, J.; Steen, H.; Roepstorff, P. Using Optimized Collision Energies and High Resolution, High Accuracy Fragment Ion Selection to Improve Glycopeptide Detection by Precursor Ion Scanning. J. Am. Soc. Mass Spectrom. 2003, 14, 777-784.

48. Warnock, D.; Bai, X.; Autote, K.; Gonzales, J.; Kinealy, K.; Yan, B.; Qian, J.; Stevenson, T.; Zopf, D.; Bayer, R. J. In Vitro Galactosylation of Human IgG at $1 \mathrm{~kg}$ Scale Using Recombinant Galactosyltransferase. Biotechnol. Bioeng. 2005, 92, 831-842.

49. Strome, S. E.; Sausville, E. A.; Mann, D. A Mechanistic Perspective of Monoclonal Antibodies in Cancer Therapy Beyond Target-Related Effects. Oncologist 2007, 12, 1084-1095.

50. Karagiannis, P.; Singer, J.; Hunt, J.; Gan, S. K.; Rudman, S. M. Mechtcheriakova, D.; Knittelfelder, R.; Daniels, T. R.; Hobson, P. S. Beavil, A. J.; Spicer, J.; Nestle, F. O.; Penichet, M. L.; Gould, H. J.; Jensen-Jarolim, E.; Karagiannis, S. N. Characterization of an Engineered Trastuzumab IgE Antibody and Effector Cell Mechanisms Targeting HER2/Neu-Positive Tumor Cells. Cancer Immunol. Immunother. 2009, 58 , 915-930. 